\title{
Silicon Nanowire Arrays for the Preconcentration and Joule Heating-Based Desorption of Trace Vapors
}

\author{
Pehr E. Pehrsson, Braden C. Giordano, Daniel C. Ratchford, Kevin Johnson
}

Chemistry Division, Naval Research Laboratory, Washington, D.C. USA

\begin{abstract}
We preconcentrated trace nitroaromatic vapors onto silicon nanowire (SiNW) arrays and desorbed them by Joule heating the array. The ordered arrays were fabricated by nanosphere lithography (NSL) combined with metal-assisted chemical etching (MACE). The nanowires had diameters of $\sim 350 \mathrm{~nm}$, lengths of $\sim 5 \mu \mathrm{m}$, and center-to-center spacing of $500 \mathrm{~nm}$. Each SiNW array chip was approximately $10^{8}$ individual wires. The nanowires were Joule heated by passing a current along their lengths, and the nanowire temperature was measured using the shift of the Si Raman single phonon line. The adsorption and selective desorption of 2,4-dinitrotoluene (2,4-DNT) and similar trace vapors was evaluated by coupling the array to a mass spectrometer.
\end{abstract}

Key words: Preconcentrator, silicon nanowire array, Joule heating, explosives

\section{Introduction}

Chemical detection with analytical instruments such as GC-MS is robust, but suffers from size, weight, power, and complexity limitations. State of the art instruments incorporate vapor sampling onto a sorbent, followed by thermal desorption to a programmable temperature vaporization (PTV) inlet, to focus the desorbed vapor for introduction to a GC for separation. This achieves detection at environmentally relevant levels, however, analysis time can be long (minutes to hours) due to a combination of sampling time and instrument duty cycle. One modification is to directly deliver the desorbed materials from a reduced stationary phase volume within the PTV inlet to the GC, thereby increasing the sampling flow and desorption rates due to the reduction in stationary phase volume. However, this approach sacrifices selectivity and sensitivity, due to eddy diffusion as analyte migrates randomly through the sorbent, and the development of radial temperature gradients during sorbent heating. These effects reduce signal to noise by orders of magnitude and cause significant errors in elution/migration time.

Here, we use uniform SiNW arrays for trace vapor concentration and selective desorption of trace vapors of DNT, TNT and related compounds to a laboratory mass spectrometer. Highly ordered SiNW arrays provide a large surface area for adsorption and reduced temperature gradients, since they are resistively heated for rapid and uniform desorption.
Custom software is used to analyze the data and will ultimately extract the maximum information from chemically complex environments.

\section{Device Fabrication and Evaluation}

Nanowire arrays (Figure 1 - top) with a porous top electrode are made by spin coating nanobeads onto a silicon substrate, followed by various wet-chemical etching and evaporation steps described more fully in [2] and [3]. The figureshows an SEM side view of the SiNW array. The porous top electrode allows analytes to pass quickly into (out of) the array during sampling (desorption). The nanowires are controllably heated by passing a current through them, which locally heats them. The SiNW temperature (versus applied current) is measured from the shift of the Si Raman single phonon line (Figure 1 - middle).

\section{Adsorption and Desorption}

Arrays were evaluated by delivering mixtures of trace TNT, DNT, and other vapors to SiNW arrays using a custom vapor handling system, at a fixed concentration, flow rate, and duration. Briefly, a calibrated permeation tube of the analyte was placed in a permeation oven and operated per manufacturers specifications to produce a nominal analyte mass flux per unit time. The volume flow rate of air through the permeation oven was $1 \mathrm{~L} / \mathrm{min}$. The nominal vapor concentration was attenuated by controlled flow of purified air, allowing for total flow rates of 3.5 to $21 \mathrm{~L} / \mathrm{min}$ or vapor 
concentration range from 28.4 to $4.7 \mathrm{ppb}_{\mathrm{v}}$. The analyte was then desorbed using a custom LabVIEW program and Keithley 2602A SourceMeter.
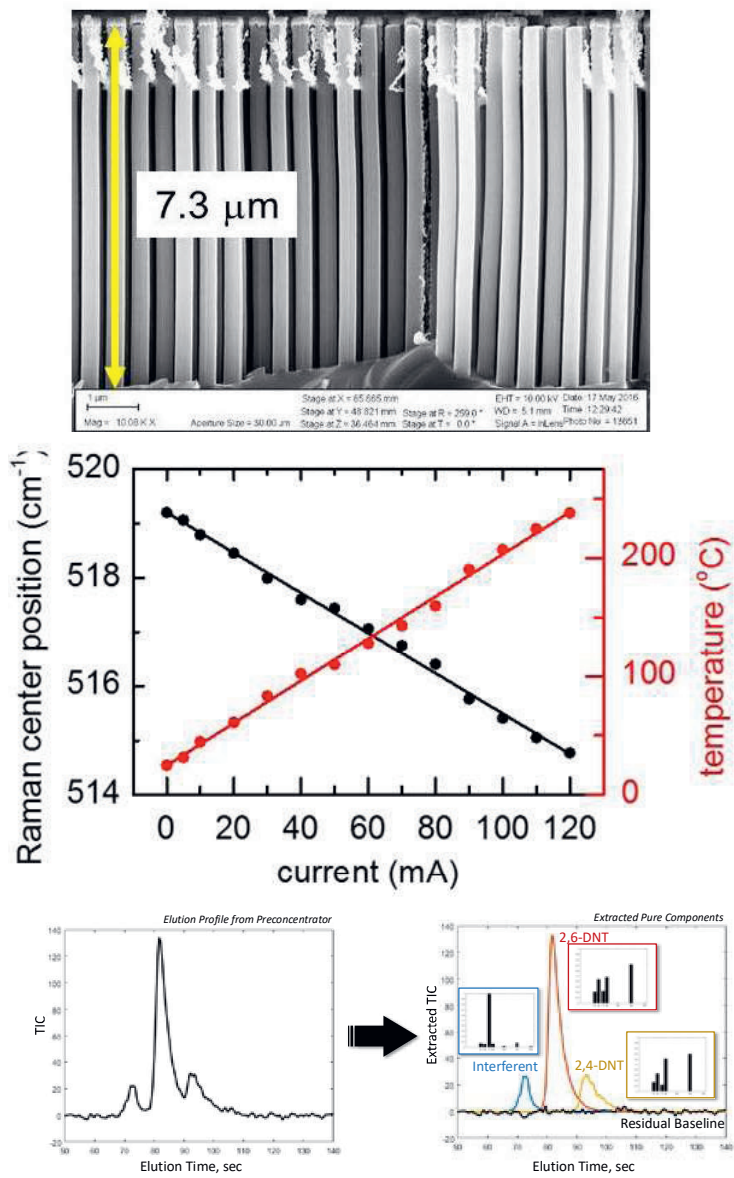

Figure 1: (top) SEM of nanowire sidewalls [1]; (middle) nanowire temperature vs. applied current [1]; (bottom) Mass spectrometer peak intensity for a mixture of 2 isomers of DNT.

Desorption from the array was detected using an Agilent 5976 mass selective detector (MSD). Briefly, the sample chamber contained a stainless steel base and a ZIF socket to which the SiNW array, mounted on a pin grid array chip, was connected. A Plexiglas or stainless steel top was sealed directly to the pin grid array chip. Multiple access ports allowed the introduction and exit of vapor, and access to the MSD by a heated capillary transfer line.

When coupled to a multichannel detector such as a mass spectrometer, it was possible to resolve multiple components by leveraging unique detector responses and the partial separation afforded by the vapor pressure of the analyte and its affinity for the array substrate. Trace vapors were detected at two orders of magnitude below their saturated vapor concentration.
Figure 1 (bottom) shows a representative desorption "chromatogram" of a mixture of 10 $\mathrm{ppb}_{\mathrm{v}} 2,6-\mathrm{DNT}$ and $3.4 \mathrm{ppb}_{\mathrm{v}} 2,4-\mathrm{DNT}$. The first peak is a highly volitile interferent associated with thermal degradation of the analytes The nominal load of 2,6-DNT on the array is approximately $15 \mathrm{ng}$, and the nominal load of 2,4-DNT is approximately $5 \mathrm{ng}$. A combination of evolving factor analysis and spectral similarity measurements allowed individual component spectra to be identified sequentially. A pure component spectum was then used to extract and estimate a corresponding pure component elution profile, the leading and trailing sides of which were fit to exponential decay functions. This allowed a peak deconvolution approach providing iterative extraction of signal from each component desorbed from the array.

\section{Conclusions}

This work demonstrates preconcentration and desorption of trace explosives vapors from SiNW arrays. The array's large surface array enables analyte adsorption without a stationary phase. Analyte is rapidly desorbed by Jouleheating the nanowires, suggesting the arrays can be used as an integrated preconcentration stage for portable analytical devices.

\section{References}

[1] D. Ratchford, J. Yeom, J.P. Long, P.E. Pehrsson, Influence of Inhomogeneous Porosity on Silicon Nanowire Raman Enhancement and Leaky Mode Modulated Photoluminescence, Nanoscale, 7, 4124-4133 (2015); doi: 10.1039/c4nr06329e

[2] J. Yeom, D. Ratchford, C.R. Field, T. Brintlinger, P.E. Pehrsson, Decoupling diameter and pitch in silicon nanowire arrays made by nanosphere lithography and metal-assisted chemical etching, Advanced Functional Materials, 24, 106 (2014); doi: 10.1002/adfm. 20130109

[3] Pehrsson, et al, US Patent 9422158, Issued 2016 